\title{
Perfil clínico-epidemiológico e sobrevida hospitalar dos casos de leucemia aguda em um hospital de referência em Rio Branco, Acre, 2007-2014
}

\author{
Clinical epidemiological profile and survival of hospital cases of \\ acute leukemia in a reference hospital in Rio Branco - Acre, \\ 2007-2014
}

\author{
Leonardo Assad Lomonaco' ${ }^{1}$ (D), Rosalina J. Koifman², Carmen Freire ${ }^{3}$ \\ 'Unidade de Atendimento de Alta Complexidade em Oncologia (Unacon) - Rio Branco (AC), Brasil. \\ 2Escola Nacional de Saúde Pública Sérgio Arouca, Fundação Oswaldo Cruz (FIOCRUZ) - Rio de Janeiro (RJ), Brasil. \\ ${ }^{3}$ Instituto de Investigación Biosanitaria de Granada (ibs.GRANADA) - Granada, Espanha.
}

Como citar: Lomonaco LA, Koifman RJ, Freire C. Perfil clínico-epidemiológico e sobrevida hospitalar dos casos de leucemia aguda e linfoma em um hospital de referência em Rio Branco, Acre, 2007-2014. Cad Saúde Colet, 2021; Ahead of Print. https://doi.org/10.1590/1414-462X202129030550

\section{Resumo}

Introdução: A Unidade de Assistência de Alta Complexidade em Oncologia (UNACON) permite o tratamento de leucemias agudas no Acre. Objetivo: Determinar o perfil clínico-epidemiológico e a sobrevida hospitalar de leucemias agudas tratadas na UNACON/Acre entre 2007 e 2014. Método: É um estudo longitudinal e retrospectivo de pacientes com leucemias agudas entre 15/06/2007 e 31/12/2014, cujos prontuários médicos forneceram dados para a análise descritiva das variáveis e posterior análise de sobrevida acumulada em 1 ano e 2 anos (método Kaplan-Meier) e comparação das curvas de sobrevida (teste de log-rank). Resultados: A sobrevida para leucemias mieloides agudas (LMA) foi de 30\% e 32\% em 1 e 2 anos, respectivamente, com pior sobrevida para pacientes masculinos, brancos, $\geq 20$ anos de idade, leucometria $<20.000$ células $/ \mathrm{mm}^{3}$, desidrogenase lática $\geq 600 \mathrm{U} / \mathrm{dl}$ e subtipo diferente do $\mathrm{M} 3$. Para leucemias linfoides agudas (LLA), a sobrevida foi de 59\% e 45\% em 1 e 2 anos, respectivamente, com pior sobrevida para sexo feminino, $\geq 20$ anos de idade e leucometria elevada. Em pacientes abaixo de 20 anos de idade com LLA, a melhor sobrevida foi observada na faixa etária de 2 a 9 anos. Conclusão: Trata-se do primeiro estudo epidemiológico de sobrevida realizado no Acre para leucemias agudas com resultados coerentes com a literatura. Contudo, novas pesquisas deverão ser realizadas. Palavras-chave: leucemias agudas; sobrevida hospitalar; Kaplan-Meier; Acre.

\begin{abstract}
Background: The High Complexity Oncology Unit (Unidade de Assistência de Alta Complexidade em Oncologia - UNACON/Acre) allowed the treatment of acute leukemias in Acre. Objective: To determine the clinical-epidemiological profile and hospital survival of acute leukemias treated at UNACON/Acre between 2007 and 2014. Method: This is a longitudinal, retrospective study of patients with acute leukemias between 06/15/2007 and 12/31/2014 whose medical records provided data for descriptive analysis of the variables, and subsequent analysis of 1-year and 2-year cumulative survival (Kaplan Meier method) and comparison of survival curves (log-rank test). Results: The survival for acute myeloid leukemia (AML) was 30 and 32\% at 1 and 2 years, respectively, with a worse survival rate for males, white,
\end{abstract}

Este é um artigo publicado em acesso aberto (Open Access) sob a licença Creative Commons Attribution, que permite uso, distribuição e reprodução em qualquer meio, sem restrições desde que o trabalho original seja corretamente citado.
Trabalho realizado na Unidade de Atendimento de Alta Complexidade em Oncologia (Unacon) - Rio Branco (AC), Brasil. Correspondência: Leonardo Assad Lomonaco. E-mail: leonardoalomonaco1@gmail.com

Fonte de financiamento: nenhuma.

Conflito de interesses: nada a declarar.

Recebido em: Nov. 27, 2018. Aprovado em: Jul. 20, 2020 
age $\geq 20$ years, leukometry $<20,000$ cells $/ \mathrm{mm}^{3}$, lactic dehydrogenase $\geq 600 \mathrm{U} / \mathrm{dl}$ and subtype different from M3. For acute lymphoid leukemias (ALL), survival was 59 and $45 \%$ at 1 and 2 years, respectively. Female gender, age $\geq 20$ years, and high leukometry had worse survival. For patients $<20$ years with $A L L$, better survival was observed in the age group of 2-9 years. Conclusion: This is the first epidemiological study of survival in Acre for acute leukemias with results consistent with the literature. However, new studies should be performed.

Keywords: acute leukemias; hospital survival; Kaplan-Meier; Acre.

\section{INTRODUÇÃO}

Leucemias agudas são neoplasias malignas hematológicas decorrentes de alterações citogenéticas adquiridas, levando a um bloqueio da hematopoese, com repercussões sistêmicas importantes. São doenças de distribuição mundial, porém a epidemiologia pode sofrer variações relacionadas às características pertinentes ao subtipo de neoplasia. Elas se dividem em dois grandes grupos: as leucemias mieloides agudas (LMA) e as leucemias linfoides agudas (LLA), com características próprias e sobrevida e prognósticos distintos ${ }^{1-6}$. Daí a importância dos estudos de sobrevida, que mostram como é o comportamento da doença à luz dos tratamentos empregados ${ }^{2,6-9}$. Assim, as pesquisas de sobrevida hospitalar acabam por apresentar uma melhor seleção dos casos e de suas características, já que a população estudada é mais uniforme em comparação às pesquisas de sobrevida populacional.

Os estudos de sobrevida para LLA realizados em diferentes países mostram estatísticas bem parecidas. Pesquisas de sobrevida populacional indicam uma sobrevida infantil em torno de $80 \% \%^{5-7,10}$, evidenciando uma mudança do curso natural dessa doença na população mais jovem. Por sua vez, trabalhos de sobrevida hospitalar mostram valores de sobrevida semelhantes e identificam subgrupos com melhor evolução, particularmente crianças menores de 10 anos, além de fatores prognósticos, como idade, leucometria e alterações citogenéticas ${ }^{1,5,7}$. Já os estudos de sobrevida populacional para LLA em adultos apresentam probabilidades de sobrevida distintas ${ }^{10-12}$. Valores de sobrevida em torno de 40 a $50 \%$ são comuns em adultos e com valores similares em sobrevida hospitalar, mostrando que esse grupo etário possui evolução mais reservada ${ }^{6,7,13}$. Nesse sentido, estudos brasileiros têm relatado valores de sobrevida hospitalar e populacional em indivíduos jovens ( $<20$ anos) com LLA que variam entre 60 a $70 \%$, enquanto em indivíduos adultos ( $>20$ anos) a sobrevida está em torno de 40 a $50 \%$.

No que se refere à LMA, a probabilidade de sobrevida continua desafiadora. Pesquisas de sobrevida populacional mostram probabilidades de sobrevida inferiores a $30 \%$, salvo alguns subtipos, como a leucemia promielocítica aguda (LPA ou LMA-M3) e a LMA CBF (core binding factor) positivas ${ }^{14-17}$. Estudos brasileiros demonstraram sobrevida hospitalar ruim para LMA ${ }^{1,2,8,18}$, com sobrevida similar à descrita nos trabalhos internacionais ${ }^{4,12,19,20}$. Callera et al. ${ }^{9}$, em São Paulo, observaram sobrevida hospitalar de 5 anos em 11,7\% dos casos de LMA; Bittencourt et al..21, no Rio Grande do Sul, relataram uma sobrevida hospitalar de 56 meses em 37\% dos casos; e Souto et al. ${ }^{22}$, no Rio de Janeiro, em uma série de casos no período de 2000 a 2008, relataram uma sobrevida hospitalar de 5 anos em $26 \%$ dos casos. No entanto, pesquisas de sobrevida hospitalar também evidenciaram que as alterações citogenéticas que ocorrem nesses casos são o principal fator prognóstico na sobrevida, como observado na LPA e na LMA tipo CBF positiva ${ }^{23,24}$.

Na região Norte do Brasil, em especial no estado do Acre, não há relatos acerca da epidemiologia dessas doenças e avaliação de sobrevida, mesmo que populacional. A descrição de casos de leucemias agudas na região, trazendo à tona suas características clínicas e epidemiológicas, além da possibilidade de se observar sua evolução e os fatores relacionados a ela, contribuem para melhorar a sobrevida dos pacientes com essas patologias. No Acre, a Unidade de Assistência de Alta Complexidade em Oncologia (UNACON), inaugurada em 2007, é referência no atendimento e tratamento dos pacientes com doenças oncológicas e onco-hematológicas em todo o estado. Nesse contexto, este estudo tem como objetivo estimar a sobrevida global dos casos de leucemias agudas tratados na UNACON/Acre, diagnosticados entre 2007 e 2014, e os possíveis fatores associados à sobrevida. 


\section{MÉTODO}

\section{População de estudo}

Foi realizado um estudo longitudinal, com coleta de dados retrospectiva, que avaliou a sobrevida global da coorte hospitalar dos pacientes de leucemia aguda, adultos e crianças, tratados integralmente na UNACON/Acre entre junho de 2007 - quando a unidade iniciou suas atividades - e dezembro de 2014. Pacientes que receberam tratamento prévio em outros centros foram excluídos da análise.

\section{Coleta de dados e variáveis de estudo}

Foi realizada a revisão dos prontuários hospitalares dos pacientes que foram matriculados e tratados integralmente na UNACON no período de 15 de junho de 2007 a 31 de dezembro de 2014. Dados também foram coletados do sistema de informatização HOSPUB, que possibilita a armazenagem das informações dos pacientes e sua verificação. Foram coletadas informações sociodemográficas, do subtipo de leucemia e valores dos parâmetros laboratoriais no diagnóstico.

No presente estudo, as variáveis foram divididas em características epidemiológicas, como sexo, idade, cor da pele e naturalidade, e clínicas, como subtipo de leucemia e valores de leucometria, plaquetometria, hemoglobina, desidrogenase lática (LDH), transaminase glutâmico-oxalacética (TGO) e transaminase glutâmico-pirúvica (TGP). Como a idade é fator prognóstico clássico nos estudos de sobrevida de leucemias agudas, ela foi dividida em três faixas etárias: de 0 a 9 anos, de 10 a 19 anos e $\geq 20$ anos. Para mostrar o impacto da idade na sobrevida para LLA, pacientes com idade inferior a 20 anos foram também subdivididos em três subgrupos: $<2$ anos, de 2 a 9 anos e de 10 a 19 anos. A variável naturalidade foi categorizada em: capital Rio Branco e municípios do interior do estado. A cor da pele foi dicotomizada em: branca e outras (parda, indígena ou amarela). Os subtipos de leucemia aguda foram agrupados em LLA e LMA, por se tratarem de doenças diferentes. A partir da informação de imunofenotipagem, os casos de LMA foram classificados no subtipo LMA-M3 e outros subtipos, e os casos de LLA, nos subtipos T e B. Com relação aos parâmetros laboratoriais, na análise de sobrevida foram utilizadas a leucometria, categorizada de acordo com o valor aproximado da mediana na amostra de casos estudada (20.000 células $/ \mathrm{mm}^{3}$ ), e a dosagem de LDH, categorizada também de acordo com a mediana observada $(600 \mathrm{U} / \mathrm{dL})$.

\section{Análise estatística dos dados}

A descrição das variáveis de estudo deu-se por meio de frequências relativas e absolutas, além de medidas de tendência central (média e mediana). $O$ teste de qui-quadrado $\left(X^{2}\right)$ avaliou as diferenças na distribuição de frequências das variáveis de estudo. A probabilidade acumulada de sobrevida global foi estimada pelo método de Kaplan-Meier e pelo teste de log-rank, sendo comparadas as curvas de sobrevida em função do sexo, cor da pele, faixa etária, subtipo de leucemia, leucometria e LDH no diagnóstico. A escolha dessas variáveis ocorreu por serem condições que se relacionam com a sobrevida de leucemias e por já terem sido empregadas em vários estudos de sobrevida ${ }^{2-6,8,10,25}$. Na análise de sobrevida, a data do diagnóstico foi considerada como o início do seguimento; os óbitos decorrentes de leucemia aguda ou qualquer outra causa foram considerados como falhas; e a censura foi a data do último contato (perda de seguimento) ou a data final do seguimento para os pacientes vivos ao final do período de estudo. Foi estimada a sobrevida acumulada após 1 ano para os pacientes diagnosticados entre 15/06/2007 e 31/12/2014, e a sobrevida acumulada após 1 e 2 anos para os pacientes diagnosticados entre 15/06/2007 e 31/12/2013.

Foi adotado nível de significância igual a 0,05 em todas as comparações. A construção do banco de dados e a análise estatística foram realizadas pelo programa SPSS Statistic Data 20.0 (Statistical Package for the Social Sciences, Chicago, IL, EUA, 2008). 


\section{RESULTADOS}

Durante o período de 15/06/2007 a 31/12/2014, foram tratados na UNACON 100 casos de leucemia aguda, sendo 65 com diagnóstico de LLA, 34 de LMA e 1 de leucemia bifenotípica. Do total, 11 pacientes receberam tratamento prévio em outros centros, restando 89 casos, 30 de LMA e 59 de LLA, para a análise da sobrevida.

A maioria dos casos de LMA tinha 20 anos ou mais, predominando o sexo feminino (Tabela 1). Em contrapartida, a maioria dos pacientes com LLA tinha idade inferior a 10 anos ( $x^{2} p$-valor $<0,001$ em relação à $\left.L M A\right)$, com predomínio do sexo masculino ( $x^{2} p$-valor $=0,50$ em relação à LMA). Tanto para LMA quanto para LLA, mais da metade dos pacientes era proveniente de municípios do interior do estado. Quanto à cor da pele, embora o número de informações ausentes fosse relativamente elevado, foi observada maior frequência de pacientes brancos entre os casos de LMA. Ainda para LMA, 11 pacientes (37\%) eram casos de LMA-M3, enquanto o subtipo $B$ foi o mais comum entre os pacientes com LLA (31\%). Contudo, $61 \%$ dos casos de LLA não tiveram seu subtipo qualificado por meio de imunofenotipagem, pois houve indisponibilidade do exame ocasionalmente.

A mediana da leucometria mostrou o valor de 18.600 células $/ \mathrm{mm}^{3}$ para LMA e de 15.150 células $/ \mathrm{mm}^{3}$ para LLA. A mediana de hemoglobina para LMA e LLA foi de 6,39 e 6,5 g/dL, respectivamente, enquanto a mediana da plaquetometria foi de 24.000 células $/ \mathrm{mm}^{3}$ para LMA e 34.100 células $/ \mathrm{mm}^{3}$ para LLA. A dosagem da LDH alterou-se nos dois subgrupos, mas com

Tabela 1. Características sociodemográficas e subtipo dos casos de leucemias mieloides agudas (LMA) e leucemias linfoides agudas (LLA) tratados na Unidade de Assistência de Alta Complexidade em Oncologia (UNACON), Acre, 2007-2014 ( $=89)$

\begin{tabular}{|c|c|c|c|}
\hline \multirow{2}{*}{ Variáveis } & LMA $(\mathbf{N}=30)$ & LLA (N = 59) & \multirow{2}{*}{ p-valor* } \\
\hline & N (\%) & N (\%) & \\
\hline \multicolumn{4}{|l|}{ Sexo } \\
\hline Masculino & $14(47)$ & $33(56)$ & 0,50 \\
\hline Feminino & $16(53)$ & $26(44)$ & \\
\hline \multicolumn{4}{|l|}{ Idade } \\
\hline $0-9$ anos & $3(10)$ & $29(49)$ & $<0,001$ \\
\hline 10-19 anos & $4(13)$ & $18(31)$ & \\
\hline$\geq 20$ anos & $23(77)$ & $12(20)$ & \\
\hline \multicolumn{4}{|l|}{ Cor da pele } \\
\hline Branca & $14(47)$ & $12(20)$ & 0,04 \\
\hline Outras & $12(41)$ & $30(51)$ & \\
\hline \multicolumn{4}{|l|}{ Naturalidade } \\
\hline Rio Branco & $9(30)$ & $19(32)$ & 0,99 \\
\hline Outros municípios & $16(53)$ & $33(56)$ & \\
\hline \multicolumn{4}{|l|}{ Subtipos } \\
\hline LMA - M3 & $11(37)$ & & \\
\hline Outras & $19(63)$ & & \\
\hline LLA-B & & $18(31)$ & \\
\hline LLA-T & & $5(8)$ & \\
\hline
\end{tabular}

*Qui-quadrado para comparação das distribuições entre LMA e LLA. 
valores mais elevados para LLA (mediana: $789 \mathrm{U} / \mathrm{dL}$ ) do que para LMA (mediana: $523 \mathrm{U} / \mathrm{dL}$ ). As dosagens de TGO e de TGP apresentaram médias elevadas, especialmente para LLA (TGO: 204 U/dL; TGP: 220 U/dL).

Para LMA, a probabilidade de sobrevida acumulada após 1 ano do diagnóstico foi de $30 \%$, com uma mediana de 45 dias (Tabela 2 e Figura 1), e nenhum dos fatores estudados teve impacto significativo na sobrevida. Para LLA, a mediana de sobrevida foi maior que 365 dias, com uma probabilidade de sobrevida ao final de 1 ano de $61 \%$, sendo a idade o único fator que mostrou impacto significativo na sobrevida, com os pacientes abaixo de 20 anos com sobrevida de 70\% (log-rank p-valor <0,001) (Tabela 2 e Figura 2). A sobrevida de 1 ano para ambos os subgrupos de LLA (B eT) foi similar (Tabela 2 e Figura 3). Analisando os casos jovens de LLA, foi

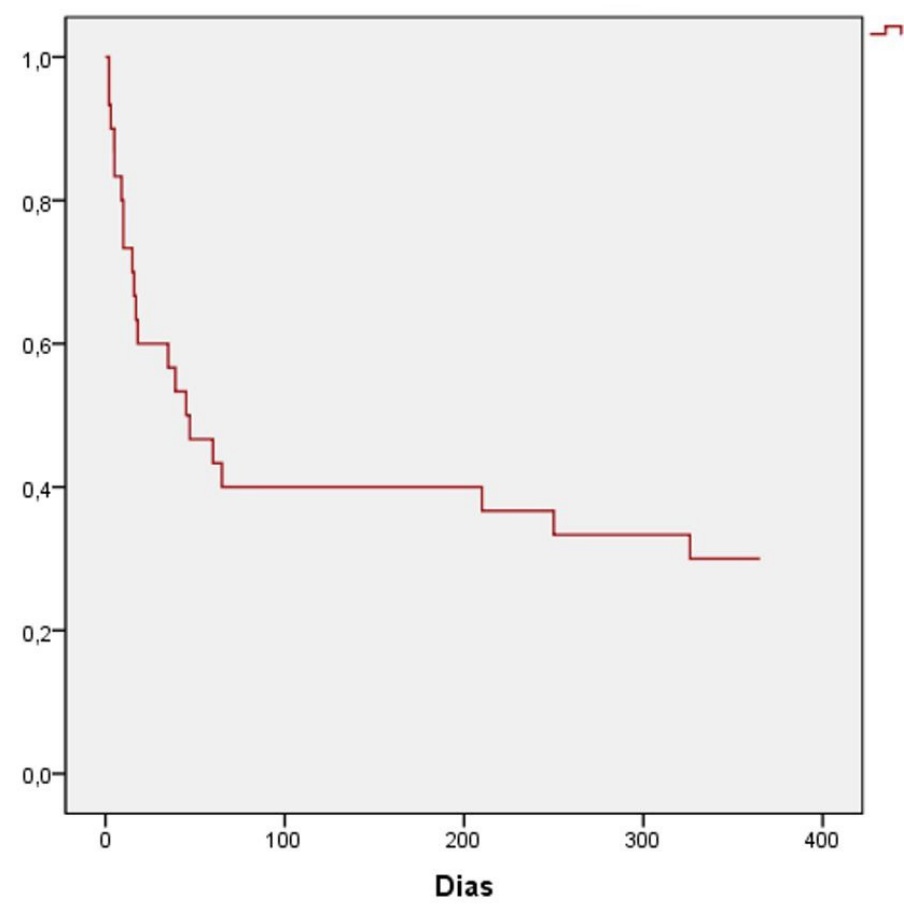

Figura 1. Sobrevida de 1 ano para os casos de leucemia mieloide aguda (LMA) (2007-2014)

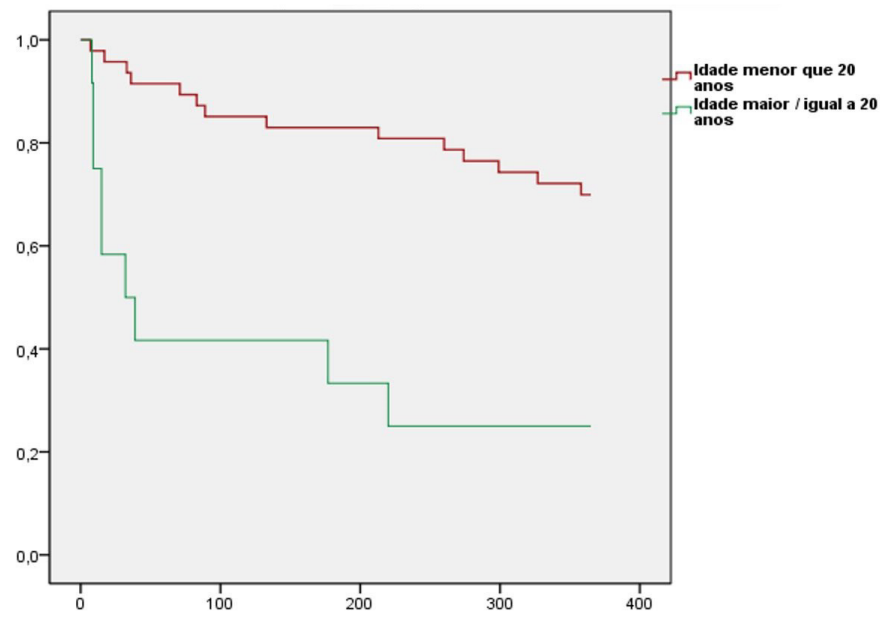

Figura 2. Sobrevida de 1 ano para os casos de leucemia linfoide aguda (LLA) de acordo com a idade (2007-2014) 


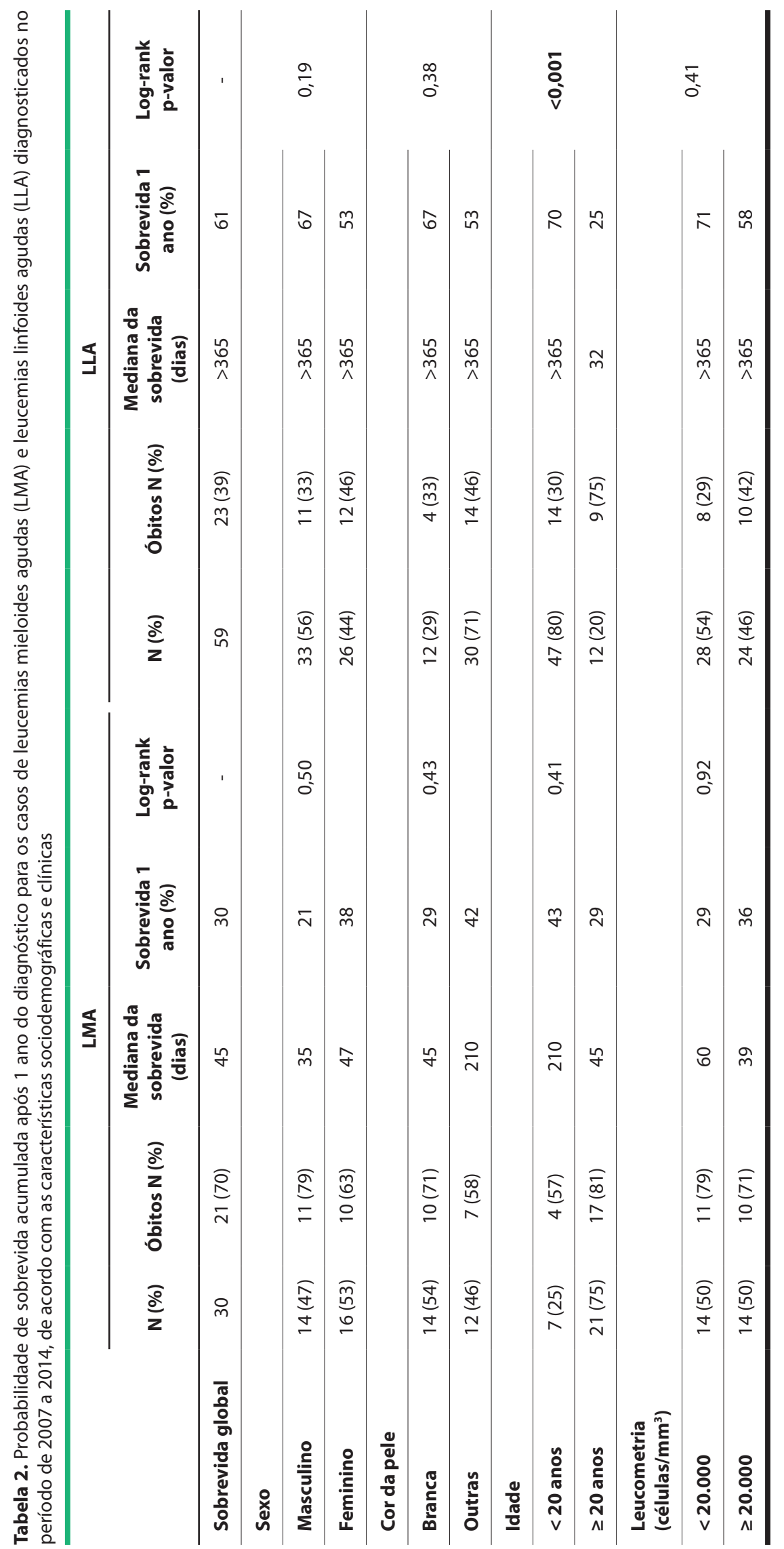




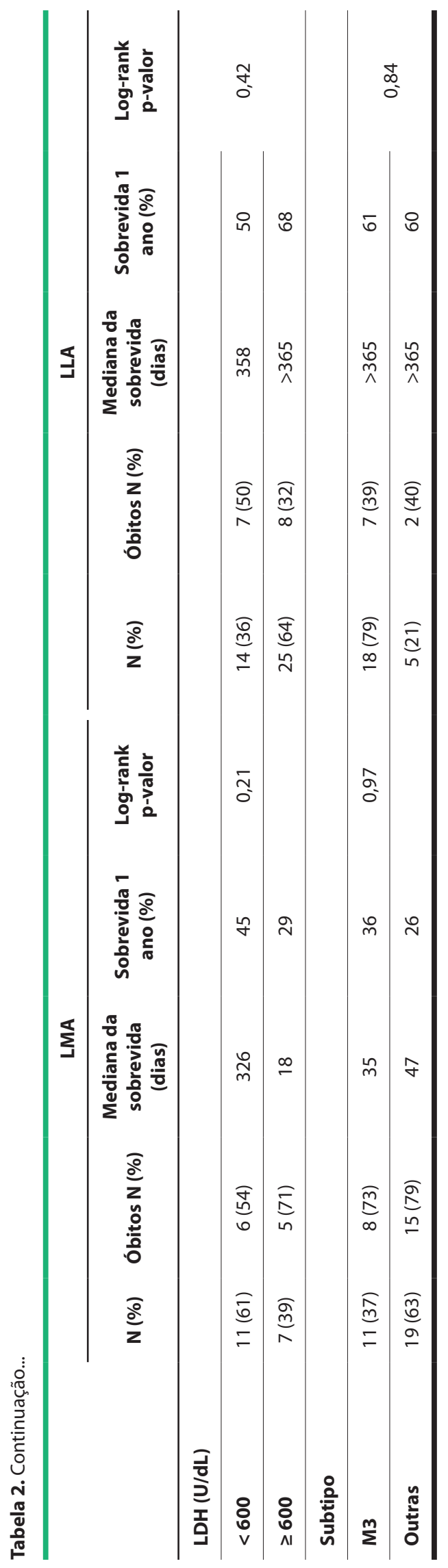


observada melhor sobrevida de 1 ano para a faixa etária de 2 a 9 anos quando comparada com a de 10-19 anos de idade ( $p$-valor $=0,02$ ) e a com menos de 2 anos ( $p$-valor $=0,01$ ) (Figura 4).

Entre 2007 e 2013, foram diagnosticados 69 casos de leucemia aguda, 25 de LMA e 44 de LLA. A distribuição das características sociodemográficas e do subtipo desses casos foi similar à descrita na Tabela 1. A sobrevida de 1 e 2 anos para esses 25 casos de LMA mostrou resultados semelhantes aos observados na coorte de casos diagnosticados entre 2007 e 2014, com probabilidade de sobrevida acumulada de 32\% e mediana de 47 dias (Tabela 3 e Figura 5). Nessa coorte, pacientes que tinham níveis menores de LDH tiveram uma maior sobrevida, porém sem alcançar significância estatística ( $p$-valor $=0,07$ ).

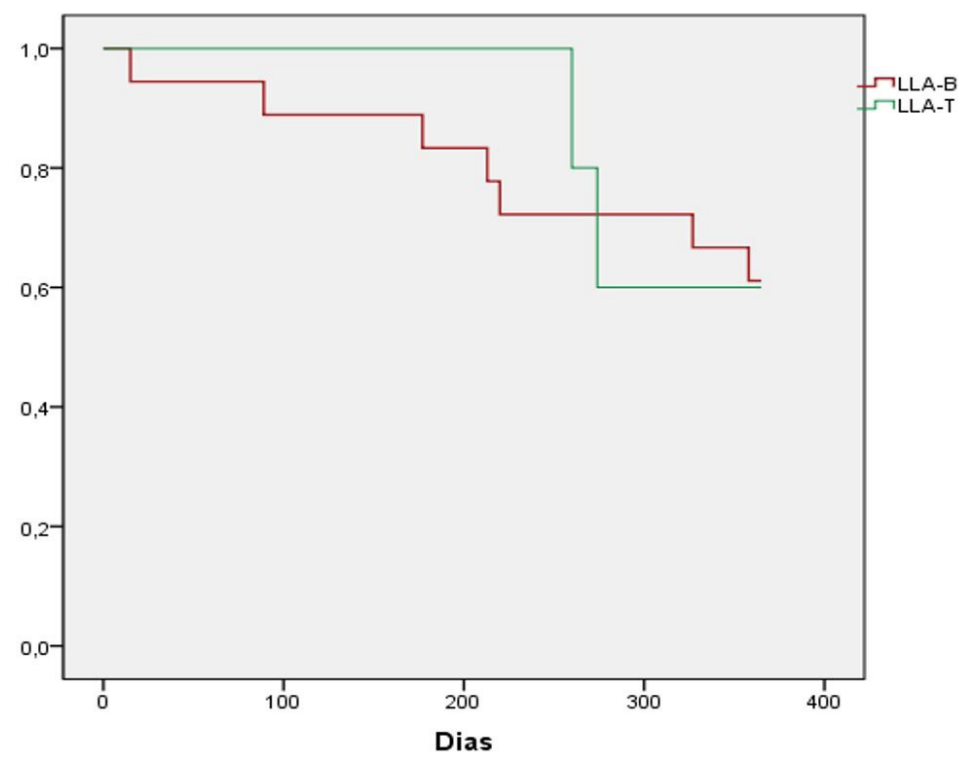

Figura 3. Sobrevida de 1 ano para os casos de leucemia linfoide aguda (LLA) de acordo com o subtipo B e T (2007-2014)

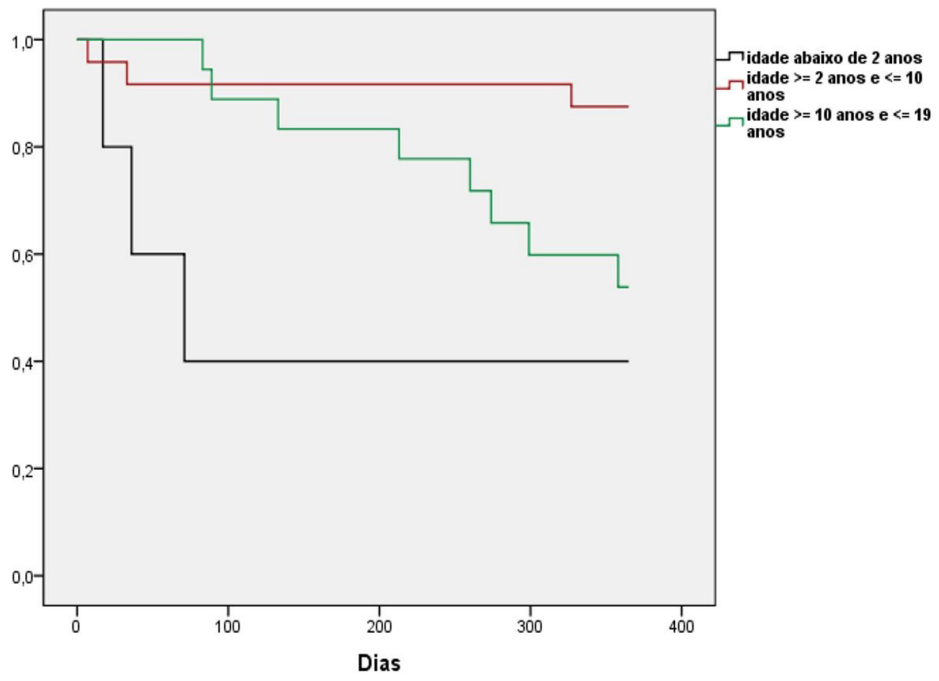

Figura 4. Sobrevida de 1 anos para os casos de leucemia linfoide aguda (LLA) em crianças e adolescentes de acordo com a idade (2007-2014) 
Para os casos de LLA diagnosticados entre 2007 e 2013, a sobrevida de 1 ano foi de 59\%, maior que a sobrevida de 2 anos, com mediana maior que 365 dias. Para sobrevida de 2 anos, a probabilidade acumulada foi de $45 \%$ e a mediana foi de 528 dias (Tabela 3). Novamente, a idade mostrou impacto significativo na sobrevida para os casos de LLA, com pacientes acima de 20 anos apresentando menor sobrevida do que pacientes jovens, tanto na análise de sobrevida de 1 ano ( $p$-valor $=0,004$ ) quanto na de 2 anos ( $p$-valor $=0,002$ ), assim como houve impacto estatístico em crianças e adolescentes (Tabela 3, Figuras 6, Figura 7). Todavia, a sobrevida de 1 ano foi pior para os pacientes com leucometria elevada do que aqueles com valores mais baixos ( $p$-valor $=0,04)$ (Tabela 3, Figura 8 .

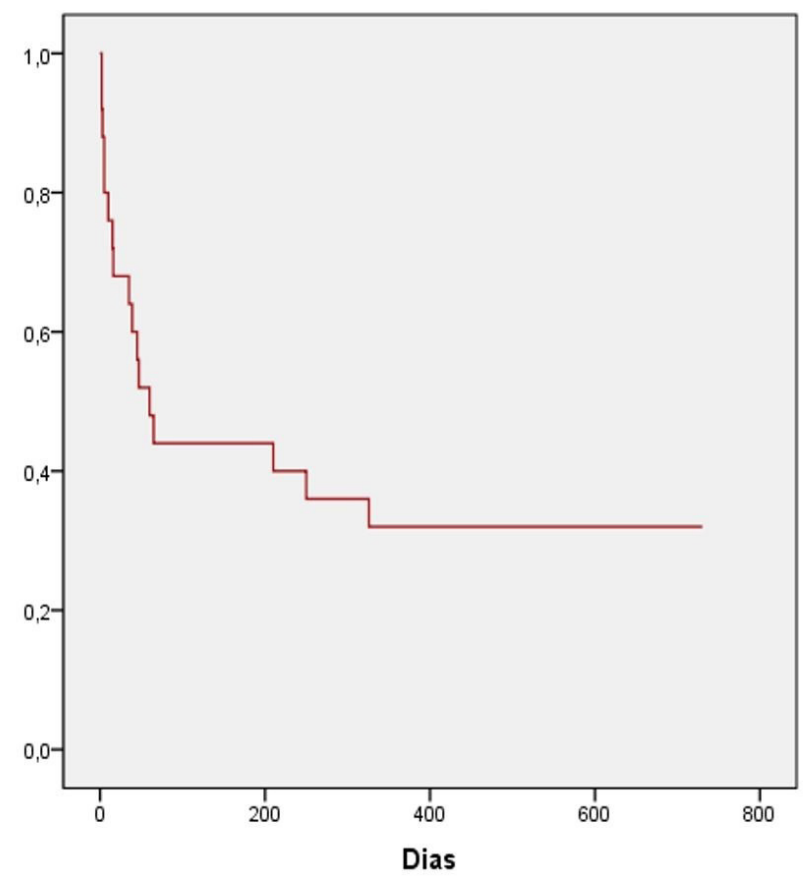

Figura 5. Sobrevida em 2 anos para os casos de leucemia mieloide aguda (LMA) (2007-2013)

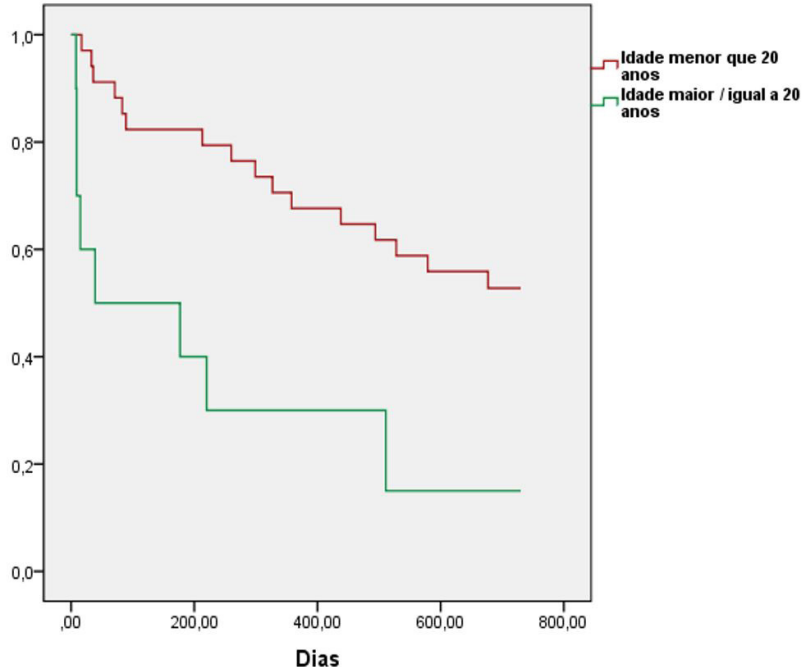

Figura 6. Sobrevida de 2 anos para os casos de leucemia linfoide aguda (LLA) de acordo com a idade (2007-2013) 


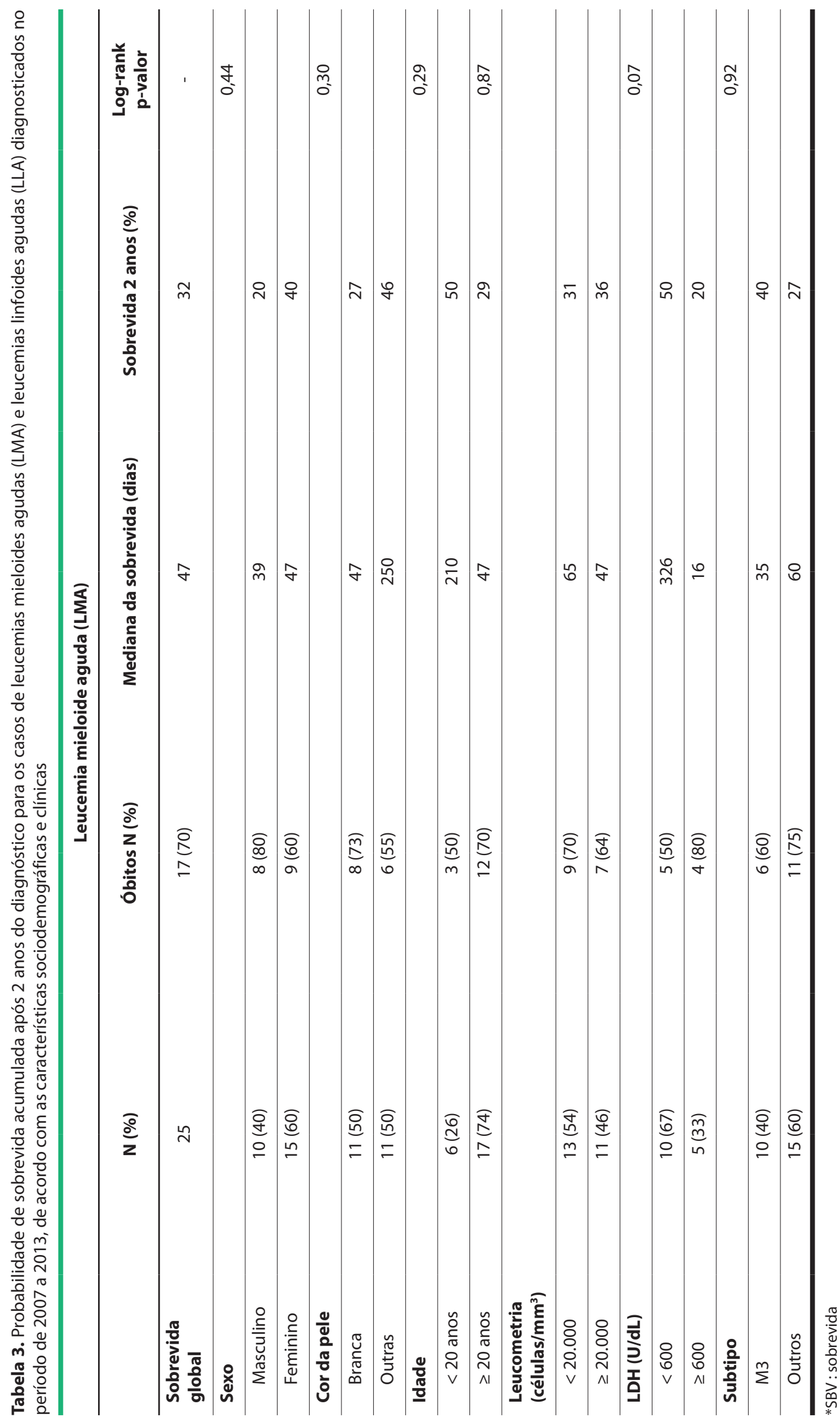




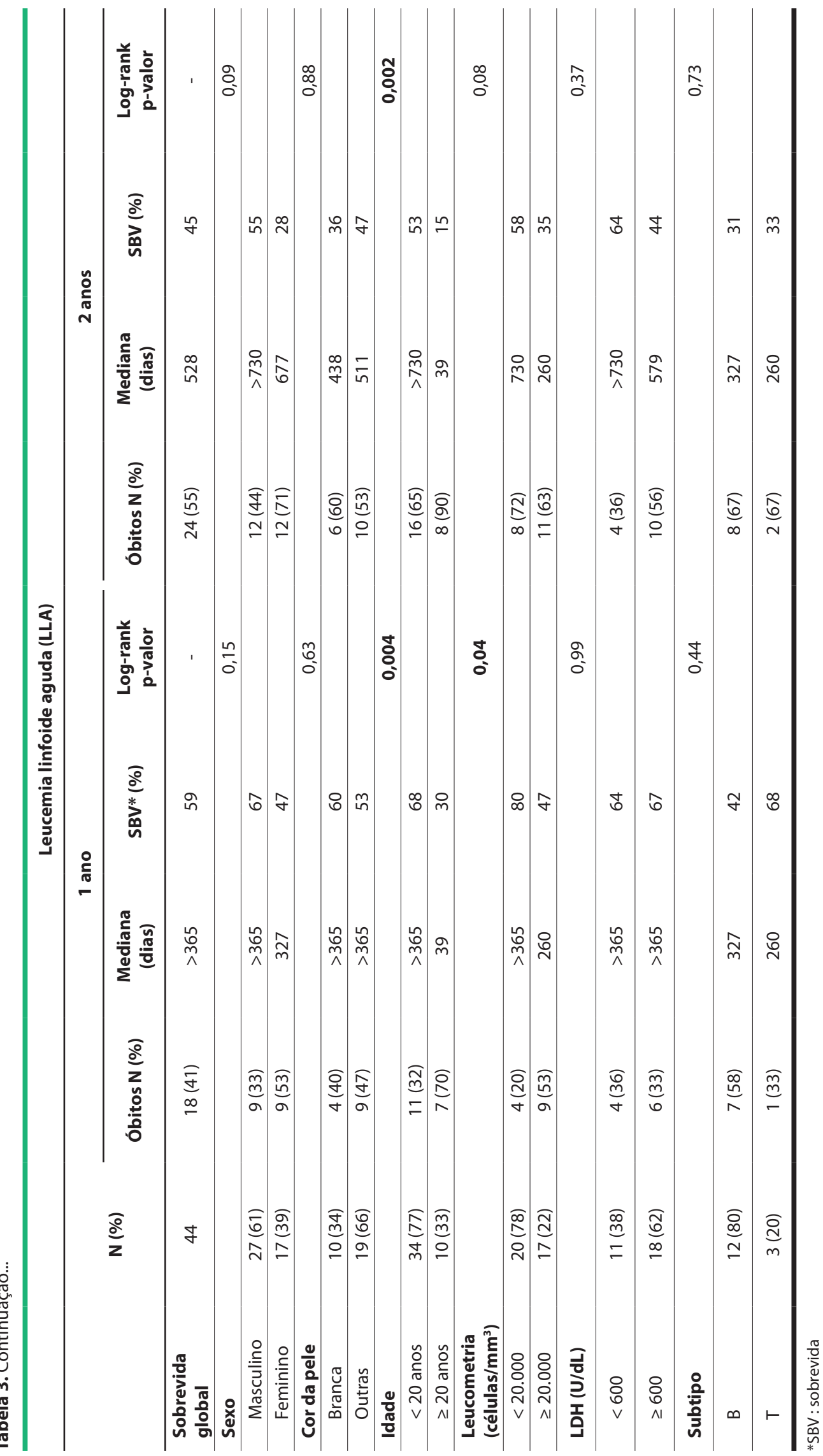




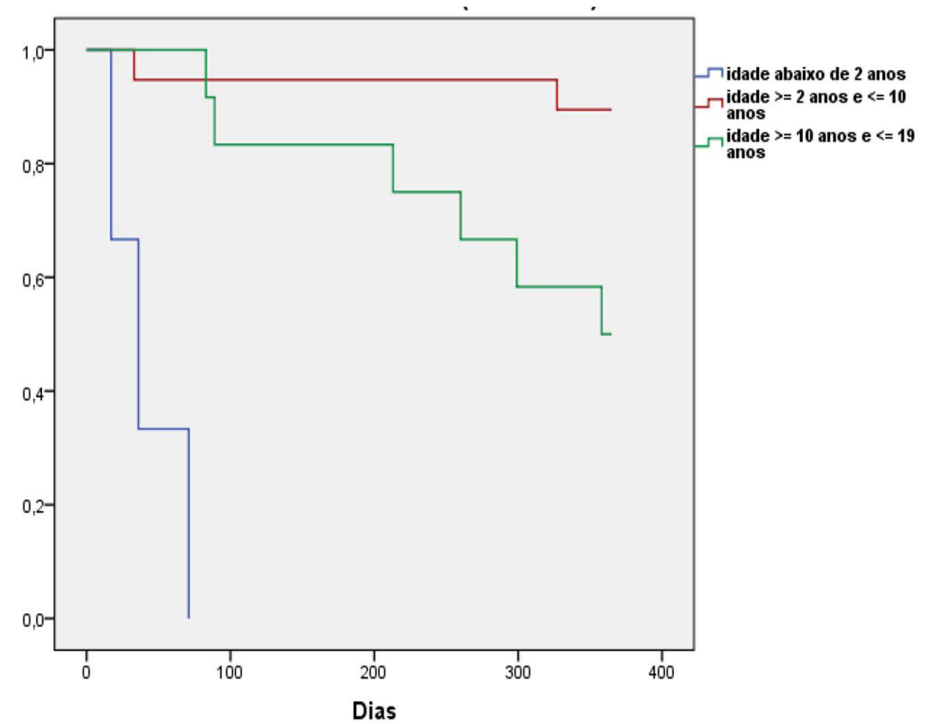

Figura 7. Sobrevida de 2 ano para os casos de leucemia linfoide aguda (LLA) em crianças abaixo e acima de 2 anos e em adolescentes (2007-2013)

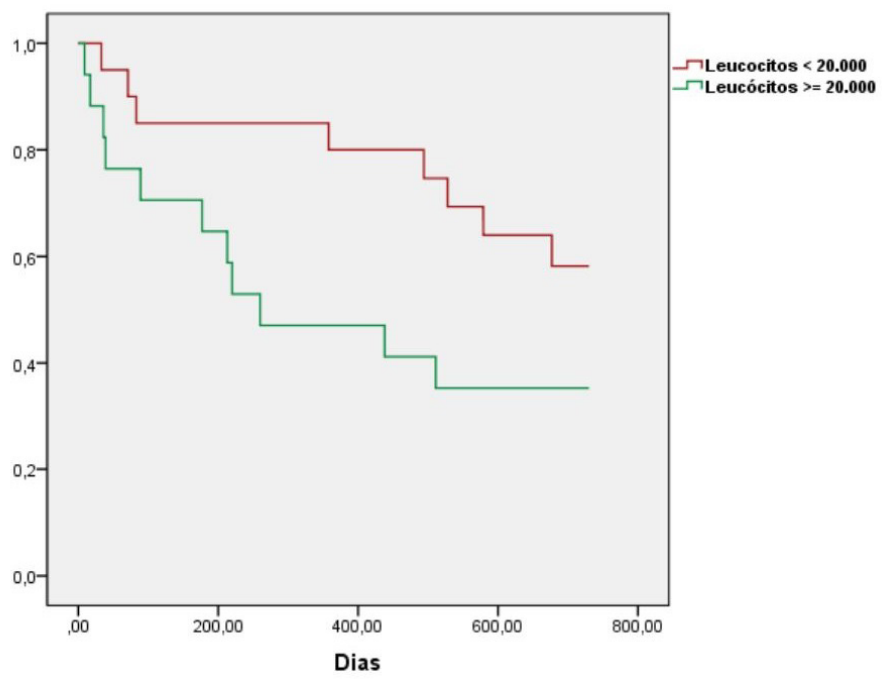

Figura 8. Sobrevida de 2 ano para os casos de leucemia linfoide aguda (LLA) de acordo com a leucometria (2007-2013)

\section{DISCUSSÃO}

Neste estudo, que avaliou a sobrevida hospitalar dos pacientes com leucemia aguda tratados em um hospital de referência no Acre, no período de 2007 a 2014, foi observada uma probabilidade de sobrevida similar aos estudos de sobrevida disponíveis na literatura: nos casos de LMA, a sobrevida de 1 e 2 anos foi de $30 \%$ e $32 \%$, respectivamente; nos casos de LLA, a sobrevida de 1 e 2 anos foi de $61 \%$ e $45 \%$ respectivamente; e os pacientes abaixo de 20 anos tiveram melhor sobrevida de 1 ano do que os pacientes acima de 20 anos (70\% e $25 \%$, respectivamente) e também em 2 anos (53\% e $15 \%$, respectivamente). A leucometria mostrou influência sobre a evolução. Nas características epidemiológicas, foi constatada a importância da idade na distribuição dos casos de leucemias agudas, bem como o predomínio 
dos subtipos LMA-M3 e LLA-B, características compartilhadas com os dados já descritos em outros estudos $1,5,8,21-23$.

A sobrevida dos casos de LMA não diferiu muito dos dados encontrados na literatura brasileira e estrangeira ${ }^{9,19,26}$. Nenhum dos fatores analisados teve impacto significativo na sobrevida de LMA, apesar de algumas observações. Níveis de LDH elevados estiveram relacionados com pior sobrevida, particularmente na sobrevida de 2 anos. Essa variável é clássica na avaliação de fatores prognósticos na sobrevida de pacientes com leucemia aguda, e valores elevados significam que há alta atividade de doença, interferindo negativamente na evolução dos paciente ${ }^{25}$. O subtipo LMA-M3 apresentou melhor sobrevida ao final de 1 ano, porém o tempo de sobrevida foi inferior ao encontrado em estudo brasileiro de sobrevida hospitalar ${ }^{23}$ e em estudos internacionais de sobrevida populacional ${ }^{15,19,26}$. Fatores como infraestrutura hospitalar e acesso limitado a serviços de saúde podem ter influenciado o desfecho desses pacientes. Outro fator relacionado à sobrevida dos casos de LMA é o valor da leucometria inicial. Leucometrias elevadas se relacionam com pior prognóstico, mas, neste estudo, leucometrias mais altas se mostraram com melhor probabilidade de sobrevida, tanto em 1 ano quanto em 2 anos. Leucometria elevada, principalmente valores acima de 100.000 células $/ \mathrm{mm}^{3}$, é descrita como fator relacionado à pior sobrevida ${ }^{8,27}$. Contudo, o valor de leucometria no diagnóstico deve ser analisado também sob outras condições, como idade e subtipos $8,25,27,28$

Para LLA, a idade foi um fator com impacto significativo na sobrevida, com melhor evolução dos pacientes jovens, particularmente na faixa etária de 2 a 9 anos (sobrevida de $88 \%)$, e sobrevida bem inferior (25\%) em pacientes com idade igual ou superior a 20 anos, o que está de acordo com a literatura ${ }^{5,29,30}$. Pacientes com LLA em faixas etárias mais altas apresentaram alterações citogenéticas de mau prognóstico, o que explicaria a sobrevida mais reservada nesse grupo. Os casos abaixo de 2 anos representaram $11 \%$ dos pacientes com LLA menores de 20 anos, e sua probabilidade de sobrevida de 1 ano foi de $40 \%$. Alterações genéticas de mau prognóstico, por exemplo, rearranjo do gene $M L L$, além de outros fatores, como infiltração do sistema nervoso central e hiperleucocitose, relacionam-se com a evolução ruim de pacientes com LLA abaixo de 2 anos $^{1,10,16,31}$. A leucometria dos pacientes com LLA também esteve relacionada à sobrevida desses casos, com valores inferiores a 20.000 células/ $\mathrm{mm}^{3}$, com melhor evolução em 1 ano. Corroborando os achados desta pesquisa, estudos anteriores demonstraram que a leucometria inicial se apresenta como fator prognóstico de sobrevida, sendo utilizada, inclusive, como critério de estratificação de risco para LLA ${ }^{5,13,32,33}$.

Os resultados encontrados no presente estudo mostram algumas características dos pacientes portadores de leucemias agudas no Acre. Até o momento, nenhum trabalho analisou a epidemiologia dessas doenças no estado, a não ser por análises de tendência de mortalidade para neoplasias malignas sólidas e incidência de câncer em Rio Branco ${ }^{34}$. Com a inauguração da UNACON em 2007, essa condição mudou, e os pacientes puderam ser tratados no Acre. Contudo, algumas limitações são encontradas. Em primeiro lugar, não foi possível incluir a totalidade de casos incidentes de leucemias agudas do estado. Nos primeiros anos de funcionamento da UNACON, muitos pacientes foram encaminhados para tratamento fora do Acre, principalmente adultos, pois pacientes pediátricos contam, desde 2007, com profissionais nessa área. Só a partir do final do ano de 2009 que as leucemias agudas começaram a ser tratadas na unidade. Assim, alguns casos não tinham sequer o registro do diagnóstico na UNACON antes de sua transferência. Esses casos eram de pacientes que foram tratados exclusivamente pelo Sistema Único de Saúde (SUS), e pacientes com maior nível socioeconômico podem ter utilizado serviços privados locais ou em outros estados. Contudo, o Acre é um estado com baixa renda per capita, em que a grande maioria da população utiliza o SUS para diagnóstico e tratamento. A UNACON é uma unidade pública de atendimento oncológico e referência para doenças oncológicas e onco-hematológicas no estado. Portanto, apesar de algumas condições impedirem o acesso a $100 \%$ dos casos incidentes, acredita-se que a maioria deles foi atendida nessa unidade.

Outras limitações foram a baixa qualidade dos registros e a falta de registro de alguns pacientes na UNACON, gerando uma procura pelos registros dos pacientes em outras clínicas e unidades para a coleta adequada dos dados e a regularização do registro na unidade. A presença de valores ausentes em algumas das variáveis analisadas pode ter influenciado alguns dos 
resultados, mas o fato de os achados terem se mostrado consistentes com a literatura sugere que vieses importantes não ocorreram.

O presente estudo avaliou as características e a evolução dos casos incidentes de leucemias agudas em uma região escassa de estudos epidemiológicos de neoplasias hematológicas e que, historicamente, apresenta restrições na área da saúde pública. Os estudos de sobrevida hospitalar, como este em questão, são o reflexo das estratégias terapêuticas empregadas contra as doenças. Não obstante, há a necessidade de que outras pesquisas sejam feitas, com intuito de aprofundar o comportamento pertinente a esses casos e seus desdobramentos em novas abordagens de conduta e tratamento no estado do Acre.

\section{REFERÊNCIAS}

1. Rego MFN, Pinheiro GS, Metze K, Lorand-Metze I. Acute leukemias in Piauí: comparison with features observed in other regions of Brazil. Braz J Med Biol Res. 2003;36(3):331-7. http://dx.doi.org/10.1590/ S0100-879X2003000300007. PMid:12640497.

2. Viana MB, Cunha KC, Ramos G, Murao M. Acute myeloid leukemia in childhood: fifteen-year experience in a single institution. J Pediatr. 2003;79(6):489-96. http://dx.doi.org/10.2223/JPED.1108. PMid:14685445.

3. Deschler B, Lübbert M. Acute myeloid leukemia: Epidemiology and etiology. Cancer. 2006 nov;107(9):2099107. http://dx.doi.org/10.1002/cncr.22233. PMid:17019734.

4. Oran B, Weisdorf DJ. Survival for older patients with acute myeloid leukemia: a population-based study. Haematologica. 2012 dez;97(12):1916-24. http://dx.doi.org/10.3324/haematol.2012.066100. PMid:22773600.

5. Leite EP, Muniz MTC, Azevedo ACAC, Souto FR, Maia ÂCL, Gondim CMF, et al. Prognostic factors in children and adolescents with Acute Lymphoblastic Leukemia. Rev Bras Saúde Mater Infant. 2007;7(4):413-21. http://dx.doi.org/10.1590/S1519-38292007000400009.

6. Stock W. Adolescents and young adults with acute lymphoblastic leukemia. ASH Educ Program Book. 2010;2010(1):21-9. http://dx.doi.org/10.1182/asheducation-2010.1.21. PMid:21239766.

7. Moorman AV, Harrison CJ, Buck GA, Richards SM, Secker-Walker LM, Martineau M, et al. Karyotype is an independent prognostic factor in adult acute lymphoblastic leukemia (ALL): analysis of cytogenetic data from patients treated on the Medical Research Council (MRC) UKALLXII/Eastern Cooperative Oncology Group (ECOG) 2993 trial. Blood. 2007;109(8):3189-97. http://dx.doi.org/10.1182/blood-2006-10-051912. PMid:17170120.

8. Padilha SL, Souza EJS, Matos MCC, Domino NR. Acute myeloid leukemia: survival analysis of patients at a university hospital of Paraná. Rev Bras Hematol Hemoter. 2015 jan;37(1):21-7. http://dx.doi.org/10.1016/j. bjhh.2014.11.008. PMid:25638763.

9. Callera F, Brasil AAV, Casali ARL, Mulin CC, Rosa ES, Barbosa MA, et al. Survival of patients diagnosed with subsets of lymphoid neoplasms and acute myeloid leukemia from 2000 to 2010 in the Vale do Paraíba, State of São Paulo: are we going the right way? Rev Bras Hematol Hemoter. 2012;34(2):168-70. http:// dx.doi.org/10.5581/1516-8484.20120038. PMid:23049408.

10. Pulte $\mathrm{D}$, Gondos A, Brenner H. Improvement in survival in younger patients with acute lymphoblastic leukemia from the 1980s to the early 21 st century. Blood. 2009;113(7):1408-11. http://dx.doi.org/10.1182/ blood-2008-06-164863. PMid:18974371.

11. Allemani C, Weir HK, Carreira H, Harewood R, Spika D, Wang X-S, et al. Global surveillance of cancer survival 1995-2009: analysis of individual data for 25676887 patients from 279 population-based registries in 67 countries (CONCORD-2). Lancet. 2015;385(9972):977-1010. http://dx.doi.org/10.1016/ S0140-6736(14)62038-9. PMid:25467588.

12. Pulte $D$, Redaniel $M T$, Jansen $L$, Brenner $H$, Jeffreys $M$. Recent trends in survival of adult patients with acute leukemia: overall improvements, but persistent and partly increasing disparity in survival of patients from minority groups. Haematologica. 2013 fev;98(2):222-9. http://dx.doi.org/10.3324/haematol.2012.063602. PMid:22929974.

13. Thomas X, Boiron JM, Huguet F, Dombret H, Bradstock K, Vey N, et al. Outcome of Treatment in Adults With Acute Lymphoblastic Leukemia: Analysis of the LALA-94 Trial. J Clin Oncol. 2004 set;22(20):4075-86. http://dx.doi.org/10.1200/JCO.2004.10.050. PMid:15353542.

14. Brunner AM, Blonquist TM, Sadrzadeh H, Perry AM, Attar EC, Amrein PC, et al. Population-based disparities in survival among patients with core-binding factor acute myeloid leukemia: a SEER database analysis. Leuk Res. 2014 jul;38(7):773-80. http://dx.doi.org/10.1016/j.leukres.2014.04.001. PMid:24793731.

15. Chen Y, Kantarjian H, Wang H, Cortes J, Ravandi F. Acute promyelocytic leukemia: A population-based study on incidence and survival in the United States, 1975-2008. Cancer. 2012 dez;118(23):5811-8. http:// dx.doi.org/10.1002/cncr.27623. PMid:22707337. 
16. Pulte D, Redaniel MT, Jansen L, Brenner H, Jeffreys M. Recent trends in survival of adult patients with acute leukemia: overall improvements, but persistent and partly increasing disparity in survival of patients from minority groups. Haematologica. 2013 fev;98(2):222-9. http://dx.doi.org/10.3324/haematol.2012.063602. PMid:22929974.

17. Shah BK, Ghimire KB. Improved survival among older acute myeloid leukemia patients: a population-based study. Acta Oncol. 2014 jul;53(7):935-8. http://dx.doi.org/10.3109/0284186X.2014.889851. PMid:24913154.

18. Zanichelli MA, Cristófani LM, Almeida MTA, Maluf PT Jr, Odone V Fo. Perspectivas para a leucemia mielóide aguda na infância após a observação de um grupo de pacientes tratados convencionalmente. Rev Bras Hematol Hemoter. 2006;28(4):246-52. http://dx.doi.org/10.1590/S1516-84842006000400006.

19. Dinmohamed AG, Visser O, van Norden Y, Blijlevens NMA, Cornelissen JJ, Huls GA, et al. Treatment, trial participation and survival in adult acute myeloid leukemia: a population-based study in the Netherlands, 1989-2012. Leukemia. 2016 jan;30(1):24-31. http://dx.doi.org/10.1038/leu.2015.188. PMid:26183645.

20. Karol SE, Coustan-Smith E, Cao X, Shurtleff SA, Raimondi SC, Choi JK, et al. Prognostic factors in children with acute myeloid leukaemia and excellent response to remission induction therapy. $\mathrm{Br} \mathrm{J}$ Haematol. 2015 jan;168(1):94-101. http://dx.doi.org/10.1111/bjh.13107. PMid:25164427.

21. Bittencourt RI, Fernandes FB, Paz AA, Fogliatto L, Astigarraga CC, Friederich JR, et al. Leucemia mielóide aguda: o olhar dos anos 2000 no Serviço de Hematologia do Hospital de Clínicas de Porto Alegre-RS. Rev Bras Hematol Hemoter. 2008;30(3):202-7. http://dx.doi.org/10.1590/S1516-84842008000300008.

22. Souto JT Fo, Portugal RD, Loureiro M, Pulcheri W, Nucci M. Characterization and analysis of the outcome of adults with acute myeloid leukemia treated in a Brazilian University hospital over three decades. Braz J Med Biol Res. 2011 jul;44(7):660-5. http://dx.doi.org/10.1590/S0100-879X2011007500058. PMid:21584439.

23. Jácomo RH, Melo RAM, Souto FR, Mattos ER, Oliveira CT, Fagundes EM, et al. Clinical features and outcomes of 134 Brazilians with acute promyelocytic leukemia who received ATRA and anthracyclines. Haematologica. 2007 out;92(10):1431-2. http://dx.doi.org/10.3324/haematol.10874. PMid:18024380.

24. Marcucci G, Mrózek K, Ruppert AS, Maharry K, Kolitz JE, Moore JO, et al. Prognostic factors and outcome of core binding factor acute myeloid leukemia patients with $\mathrm{t}(8 ; 21)$ Differ from those of patients with inv(16): a cancer and leukemia group B study. J Clin Oncol. 2005 jul;23(24):5705-17. http://dx.doi.org/10.1200/ JCO.2005.15.610. PMid:16110030.

25. Döhner H, Estey EH, Amadori S, Appelbaum FR, Büchner T, Burnett AK, et al. Diagnosis and management of acute myeloid leukemia in adults: recommendations from an international expert panel, on behalf of the European LeukemiaNet. Blood. 2010;115(3):453-74. http://dx.doi.org/10.1182/blood-2009-07-235358. PMid:19880497.

26. Sant M, Minicozzi P, Mounier M, Anderson LA, Brenner H, Holleczek B, et al. Survival for haematological malignancies in Europe between 1997 and 2008 by region and age: results of EUROCARE-5, a populationbased study. Lancet Oncol. 2014;15(9):931-42. http://dx.doi.org/10.1016/S1470-2045(14)70282-7. PMid:25030467.

27. Nguyen S, Leblanc T, Fenaux P, Witz F, Blaise D, Pigneux A, et al. A white blood cell index as the main prognostic factor in $t(8 ; 21)$ acute myeloid leukemia $(A M L)$ : a survey of 161 cases from the French $A M L$ Intergroup. Blood. 2002;99(10):3517-23. http://dx.doi.org/10.1182/blood.V99.10.3517. PMid:11986202.

28. Malkan UY, Gunes G, Eliacik E, Haznedaroglu IC, Etgul S, Aslan T, et al. The factors affecting early death after the initial therapy of acute myeloid leukemia. Int J Clin Exp Med. 2015;8(12):22564-9. PMid:26885243.

29. Ma H, Sun H, Sun X. Survival improvement by decade of patients aged 0-14 years with acute lymphoblastic leukemia: a SEER analysis. Sci Rep. 2015;4(1):4227. http://dx.doi.org/10.1038/srep04227. PMid:24572378.

30. Gatta G, Rossi S, Foschi R, Trama A, Marcos-Gragera R, Pastore G, et al. Survival and cure trends for European children, adolescents and young adults diagnosed with acute lymphoblastic leukemia from 1982 to 2002. Haematologica. 2013 maio;98(5):744-52. http://dx.doi.org/10.3324/haematol.2012.071597. PMid:23403323.

31. Ibagy A, Silva DB, Seiben J, Winneshoffer APFF, Costa TEJB, Dacoregio JS, et al. Acute Lymphoblastic Leukemia in Infants: 20 years of experience. J Pediatr. 2013 jan;89(1):64-9. http://dx.doi.org/10.1016/j. jped.2013.02.010. PMid:23544812.

32. Marks DI. Treating the "older" adult with acute lymphoblastic leukemia. ASH Educ Program Book. 2010;2010(1):13-20. http://dx.doi.org/10.1182/asheducation-2010.1.13. PMid:21239765.

33. Pui C-H, Evans WE. Treatment of Acute Lymphoblastic Leukemia. N Engl J Med. 2006 jan;354(2):166-78. http://dx.doi.org/10.1056/NEJMra052603. PMid:16407512.

34. Nakashima JP, Koifman S, Koifman RJ. Cancer mortality trends in Rio Branco, Acre State, Brazil, 19802006. Cad Saude Publica. 2011;27(6):1165-74. http://dx.doi.org/10.1590/S0102-311X2011000600013. PMid:21710013. 